\title{
Resultados preliminares do estudo da metodologia de tomografia sísmica de ruído ambiental aplicada à escala rasa.
}

\author{
Bruno Nascimento Ferreira*1, Priscila Martins Oliveira ${ }^{1}$, Marcelo Peres Rocha ${ }^{1}$, Marcos Vinícius Ferreira ${ }^{1}$, Martin Schimmel ${ }^{2}$ \\ ${ }^{1}$ Instituto de Geociências / Universidade de Brasília \\ 2Institute de Cièncias de la Terra "Jaume Almera", Espanha
}

Copyright 2014, SBGf - Sociedade Brasileira de Geofísica.

Este texto foi preparado para a apresentação no VI Smpósio Brasileiro de Geofísica, Porto Alegre, 14 a 16 de outubro de 2014. Seu conteúdo foi revisado pelo Comitê Técnico do VI SimBGf, mas não necessariamente representa a opinião da SBGf ou de seus associados. É proibida a reprodução total ou parcial deste material para propósitos comerciais sem prévia autorização da SBGf.

\begin{abstract}
In this work we present the partial results obtained with Ambiental Noise Tomography (ANT) methodology applied to an area at the campus of University of Brasília. The goal was to evaluate the validity of the method, in small scale, which is an unusual application. The results showed that there is a dependence of the recovered signal with the distance between stations. They decrease in amplitude as the distance increases, being hard to distinguish signal from noise beyond $6 \mathrm{~m}$. It was established the minimum number of stacked signals to recover acceptable traces, what can be used to reduce the time of acquisition and processing. The dispersion curves analyzed suggests a mean group velocity of the $140 \mathrm{~m} / \mathrm{s}$.
\end{abstract}

\section{Introdução}

Obter uma fonte controlada, com energia suficientemente alta para propagar um sinal detectável pelo meio, sempre representou um impasse para a aplicação dos métodos sísmicos. Alcançar uma razão sinal/ruído adequada, especialmente em regiões ruidosas, como em ambientes urbanos, requer fontes extremamente energéticas e portanto de difícil aplicabilidade em alguns casos. Neste contexto, o ruído incoerente, indesejado na sísmica convencional, surge como um aliado nos levantamentos de tomografia sísmica de ruído ambiental.

O ruído ambiental é composto por todo e qualquer movimento oscilatório do meio, gerado de modo aleatório por processos naturais, como o movimento das ondas do mar, o vento batendo em árvores, atividade tectônica, ou por ações antrópicas, como o fluxo de veículos, movimentação de pessoas, construções etc.

A tomografia sísmica de ruído ambiental (ANT) (Bensen et al., 2007) se baseia na correlação cruzada entre os registros simultâneos de duas estações sismológicas. Através da correlação cruzada obtêm-se uma estimativa da função de Green (Claerbout, 1968; Wapenaar et al., 2010), que contêm as características do meio físico que separam as duas estações, como as velocidades das ondas sísmicas em função da frequência das ondas.
Esta técnica foi aplicada inicialmente em por Shapiro et al. (2005), e tem sido bastante utilizada para estudos da crosta e do manto da Terra, com resultados satisfatórios, especialmente em áreas com alta densidade de estações sísmicas (Bensen et al., 2007). Entretanto, aplicações rasa do método são incomuns, com os primeiros trabalhos na área surgindo a menos de uma década (Nunziata et al. 2009). carecendo portanto de mais estudos. O objetivo proposto por esse trabalho é justamente avaliar a aplicação do método de tomografia de ruído ambiental em pequena escala (poucas dezenas de metros), uma vez que, conceitualmente falando, não existem restrições.

\section{Área de Estudo}

Os dados utilizados foram adquiridos em uma área localizada no campus Darcy Ribeiro da Universidade de Brasília, próximo ao Instituto de Geociências, de dimensões 10,5mx7,5m, como mostrado na Figura 1.
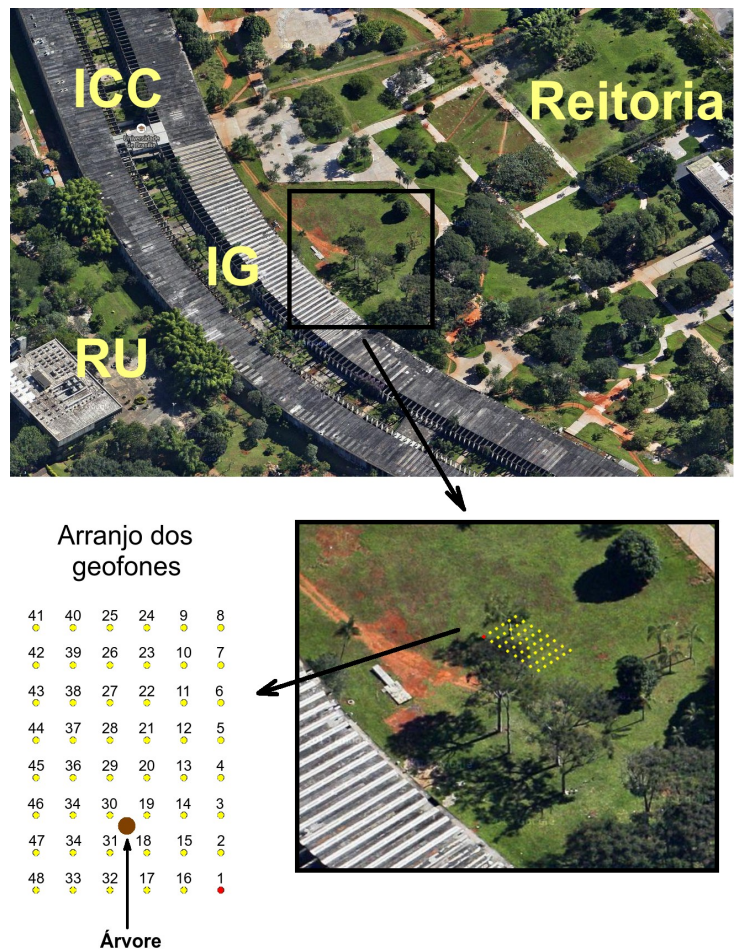

Figura 1: Local de estudo, localizado no campus Darcy Ribeiro da Universidade de Brasília atrás do Instituto Central de Ciências (ICC). A disposição dos geofones segue um arranjo regular com espaçamento de $1,5 \mathrm{~m}$, que na imagem estão representados por círculos amarelos. Legenda: IG = Instituto de Geociências, $R U=$ Restaurante Universitário. 
A área é caracterizada por ser relativamente plana, com gramíneas, e com solo do tipo latossolo vermelho característico da região, com espessura entre 5 e $7 \mathrm{~m}$, avaliada em trabalhos anteriores, com sísmica de refração (Rocha et al., 2011).

\section{Metodologia}

Para aquisição foi montado um grid retangular, de $6 \times 8$ geofones, espaçados de $1,5 \mathrm{~m}$ cada. A disposição dos geofones foi feita de modo a incluir uma árvore de grande porte localizada dentro da área de estudo (Figura 1). Um dos objetivo foi testar se o método poderia trazer informações a respeito da mesma, pois acredita-se haver um contraste local de velocidade das ondas sísmicas entre as raízes da árvore e o meio circundante.

A aquisição dos dados consistiu em acionar todos os geofones simultaneamente por um intervalo de $1 \mathrm{~s}$ com um gatilho manual, sem fonte. Deu-se o nome de "medida", a cada vez que esse procedimento foi repetido. Foram realizadas um total de 520 medidas. O objetivo de se obter várias medidas reside no fato da recuperação das ondas de superfície, através da correlação cruzada, ser influenciada por "ruídos indesejados", não relacionados à propagação das ondas de uma estação para a outra. Somando-se várias medidas para um dado par de estações, esse ruído pode ser minimizado (empilhamento). Existem vários tipos de empilhamento, entretanto, neste trabalho foi utilizado o empilhamento de fase, desenvolvido por Schimmel et al. (1997).

Metade dos dados foram adquiridos com a presença de um forte ruído coerente, proveniente de um gerador elétrico localizado em uma casa de máquinas a poucos metros do grid. Posteriormente tais dados se mostraram inutilizáveis, pois o ruído coerente de alta amplitude mascara completamente o sinal do ruído ambiental.

O processamento dos dados pós aquisição consistiu em realizar a correlação cruzada entre cada par de geofones, levando-se em conta que a correlação referente ao par $x-y$ é igual a correlação do par $y-x$, ou seja, é feita a consideração que o meio é isotrópico com relação a passagem da ondas. Essa consideração de modo geral não é válida, mas como a região tinha um solo espesso, e o caráter anisotrópico se torna mais aparente em rochas, ela foi adotada .Para a correlação entre os pares de estações foi utilizada a técnica de Correlação Cruzada de Fase (PCC em inglês) desenvolvida por Schimmel (1999).

Foram computadas ao todo 586.560 correlações, e 1.128 traços empilhados. A equação (1) fornece o número total de correlações em função do número de geofones e de medidas realizadas.

$$
\mathrm{N}_{\mathrm{c}}=\left(\mathrm{N}_{\mathrm{g}}-1\right) \cdot\left(\mathrm{N}_{\mathrm{g}} / 2\right) \mathrm{N}_{\mathrm{m}}
$$

Onde $\mathrm{N}_{\mathrm{c}}$ é o número de correlações, $\mathrm{N}_{\mathrm{m}}$ o número de medidas e $\mathrm{N}_{\mathrm{g}}$ o número de geofones. $\mathrm{O}$ intervalo de lag das correlações foi de $-0,5$ s até $0,5 s$.
Os computadores utilizados tinham a capacidade de processar aproximadamente 6 correlações por minuto, levando cerca de 2 meses de esforço computacional para a conclusão desta etapa. O empilhamento dos 260 traços iniciais (referentes a aquisição sem o ruído proveniente do gerador elétrico) levaram cerca de $1,2 \mathrm{~h}$ de processamento para cada par de geofones, logo, aproximadamente mais 2 meses foram gastos nessa etapa.

A metodologia seguida neste trabalho pode ser sumarizada de acordo com a Figura 2.

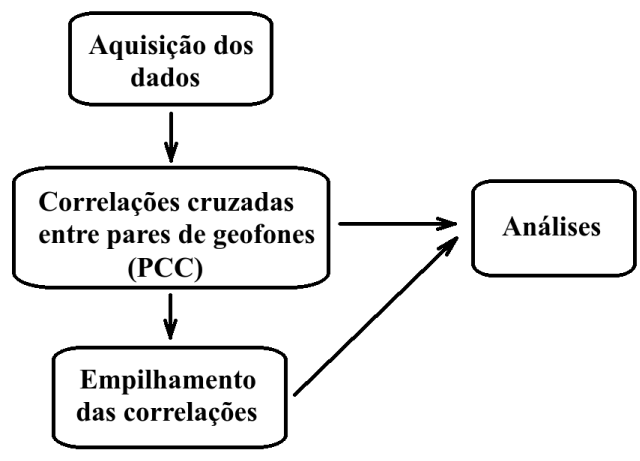

Figura 2: Fluxograma indicativo das etapas realizadas.

A Figura 3 ilustra as duas primeiras etapas do trabalho, referentes à aquisição e às correlações de todos os pares.

\begin{tabular}{|c|c|c|c|c|c|}
\hline & \multicolumn{5}{|c|}{ Etapa } \\
\hline Medida & Aquisição & \multicolumn{4}{|c|}{ Correlações cruzadas de fase (PCC) } \\
\hline 1 & $\begin{array}{c}01 \\
02 \\
03 \\
\vdots \\
48\end{array}$ & $\begin{array}{c}01 \text { com } 02 \\
01 \text { com } 03 \\
01 \text { com } 04 \\
\vdots \\
01 \text { com } 48\end{array}$ & $\begin{array}{c}02 \operatorname{com} 03 \\
02 \operatorname{com} 04 \\
02 \operatorname{com} 05 \\
\vdots \\
02 \operatorname{com} 48\end{array}$ & $\begin{array}{r}46 \operatorname{com} 47 \\
\cdots \quad 46 \operatorname{com} 48\end{array}$ & $47 \operatorname{com} 48$ \\
\hline 2 & \begin{tabular}{|c|}
01 \\
02 \\
03 \\
$\vdots$ \\
48
\end{tabular} & $\begin{array}{c}01 \text { com } 02 \\
01 \text { com } 03 \\
01 \text { com } 04 \\
\vdots \\
01 \text { com } 48\end{array}$ & $\begin{array}{c}02 \operatorname{com} 03 \\
02 \operatorname{com} 04 \\
02 \operatorname{com} 05 \\
\vdots \\
02 \operatorname{com} 48\end{array}$ & $\begin{array}{r}46 \operatorname{com} 47 \\
\ldots \quad 46 \operatorname{com} 48\end{array}$ & $47 \operatorname{com} 48$ \\
\hline \multicolumn{6}{|c|}{ • } \\
\hline 520 & $\begin{array}{c}01 \\
02 \\
03 \\
\vdots \\
48\end{array}$ & $\begin{array}{c}01 \text { com } 02 \\
01 \text { com } 03 \\
01 \text { com } 04 \\
\vdots \\
01 \text { com } 48\end{array}$ & $\begin{array}{c}02 \operatorname{com} 03 \\
02 \operatorname{com} 04 \\
02 \operatorname{com} 05 \\
\vdots \\
02 \operatorname{com} 48\end{array}$ & $\begin{array}{r}46 \operatorname{com} 47 \\
\ldots \quad 46 \operatorname{com} 48\end{array}$ & $47 \operatorname{com} 48$ \\
\hline
\end{tabular}

Figura 3: Quadro ilustrativo onde se vê o número das medidas, os traços gerados para cada geofone na aquisição, e os pares de correlação cruzada para cada medida.

\section{Resultados e Discussão}

A Figura 4 ilustra os traços obtidos com diferentes números de sinais empilhados, para o par de geofones 01-02. Percebe-se uma tendência a estabilização do sinal quanto maior o número de empilhamento, ficando 
praticamente imperceptível a diferença entre a curva de 100 sinais empilhados com a de 250 .

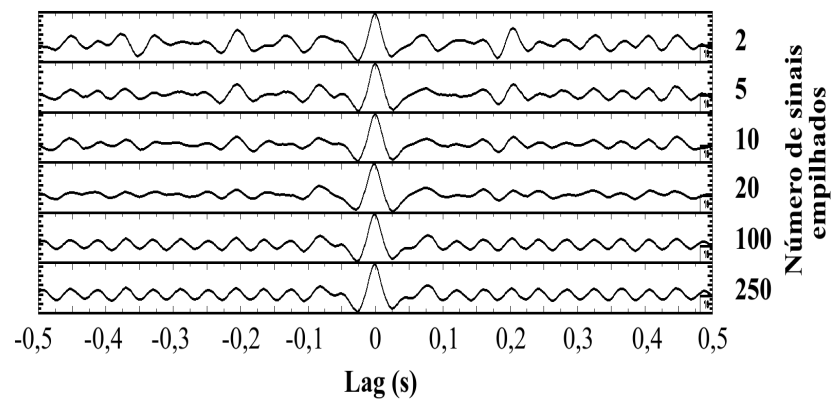

Figura 4: Sinal resultante do empilhamento de diferentes quantidades de sinais correlacionados para o par de geofones 1 e 2 .

Assume-se que o melhor sinal possível (MSP) de ser obtido com um número $\mathrm{n}$ de medidas será o empilhamento dos $\mathrm{n}$ sinais correlacionados. Nesse contexto, pode-se avaliar o quão próximo do MSP estão os sinais provenientes de empilhamentos com um número menor de medidas. Essa análise é feita através da correlação cruzada entre os empilhamentos parciais com o MSP. Ela pode estabelecer critérios para diminuir o número de medidas realizadas diminuindo o tempo em campo, e consequentemente, o tempo computacional gasto no processamento. A Figura 5 mostra esse tipo de análise feita para os pares de geofones 01-02 e 01-05.

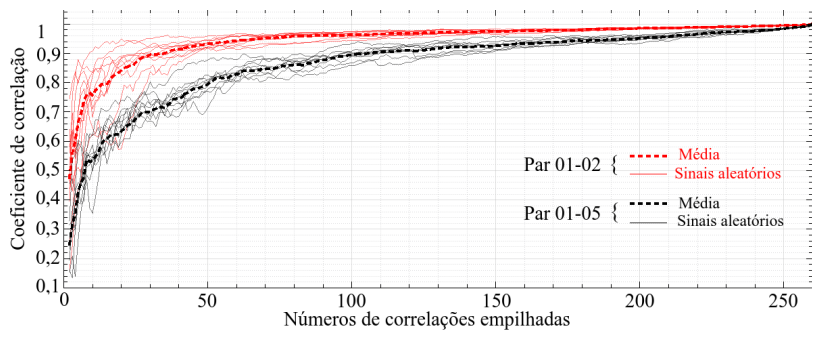

Figura 5: Gráfico mostrando a relação entre o coeficiente de correlação entre o MSP e os número de séries temporais empilhadas. Em vermelho temos o comportamento referente ao par 01-02 (mais próximos) e em preto ao par 01-05 (mais distantes).

Fica claro que a distância entre os geofones é um fator fundamental para a recuperação do sinal. Para o par de geofones 01-02, que estão separados por $1,5 \mathrm{~m}$, bastariam 30 medidas para garantir um nível de similaridade de $90 \%$ entre as curvas do melhor sinal e o empilhamento de 30 medidas. Já para o par 01-05, separados por $6 \mathrm{~m}$, seriam necessários 100 medidas para se obter o mesmo nível de similaridade. Desta forma, sugere-se que pelo menos 100 medidas sejam realizadas. Em trabalhos de escala regional, este numero ficou em torno de 60 medidas (Ferreira, 2013).

Uma análise da direção preferencial da fonte de ruído foi realizada (Figura 6). Levou-se em conta pares de geofones separados por uma mesma distância $(\sim 7,5 \mathrm{~m})$, já que pelas análises anteriores, fica claro que o sinal recuperado pelas correlações cruzadas depende da distância entre eles. De acordo com os resultados da Figura 6 observou-se que as melhores direções de recuperação dos sinais foram as relacionadas com o alinhamento dos pares de geofones 01-36 (Figura 6a) e 20-41 (Figura 6b). Segundo estes resultados, e conforme a Figura 1 é possível supor que existe uma fonte de ruído preferencial nesta direção e que coincide com o alinhamento com a Reitoria da UnB, que é um prédio com movimento significativo de pessoas e carros. Provavelmente o ICC é também uma fonte de ruídos, mas que afeta todas as direções de forma aproximadamente igual.

Foram realizadas várias tentativas de geração de curvas de dispersão para obtenção das velocidades de grupo relacionadas à ondas recuperadas com o método, mas poucas tiveram bons resultados. Para geração das curvas de dispersão foi utilizada a Técnica de Múltiplas Filtragem (MFT em inglês), desenvolvida por .

A Figura 7 é um exemplo de uma curva satisfatória. Nesta figura pode-se observar que a velocidade de grupo varia entre 130 e $170 \mathrm{~m} / \mathrm{s}$. Estes valores são razoáveis quando comparados com os valores médios para onda $P$ obtidos por Rocha et al. (2011) na mesma área $(376 \mathrm{~m} / \mathrm{s}$ para o período seco e $516 \mathrm{~m} / \mathrm{s}$ para o período chuvoso). A onda Rayleigh possui valores de velocidade menores que os da onda S, e esta pode ser estimada como $70 \%$ do valor da onda $P$.
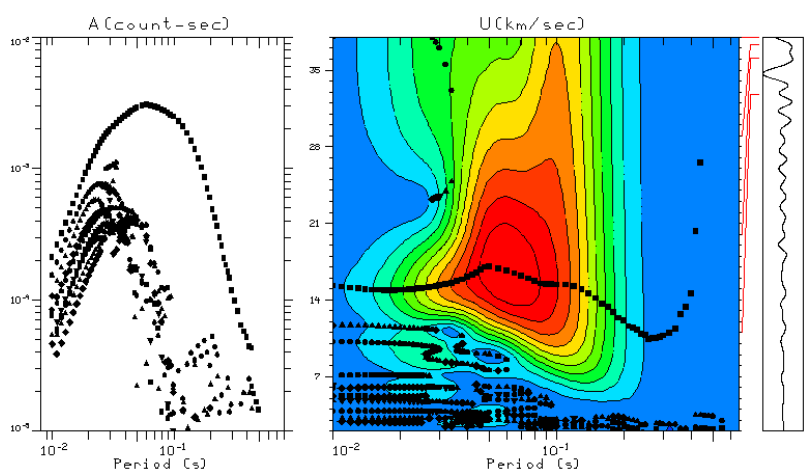

Figura 7: Curva de dispersão obtida para o par de geofones 05-48, distanciados de 10,8 metros. A escala de velocidade está multiplicada por um fator de 100 (e.g. $14 \mathrm{~km} / \mathrm{s}, 14000 \mathrm{~m} / \mathrm{s}$, refere-se a $140 \mathrm{~m} / \mathrm{s}$ ). 


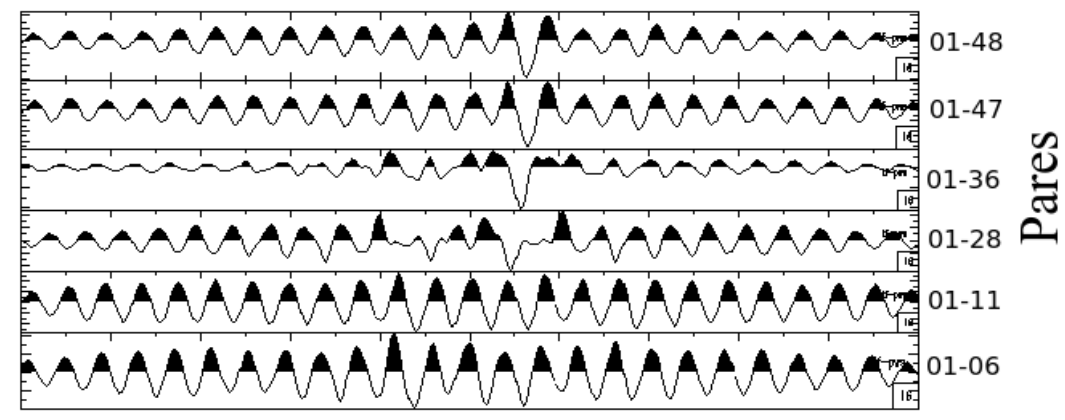

a)
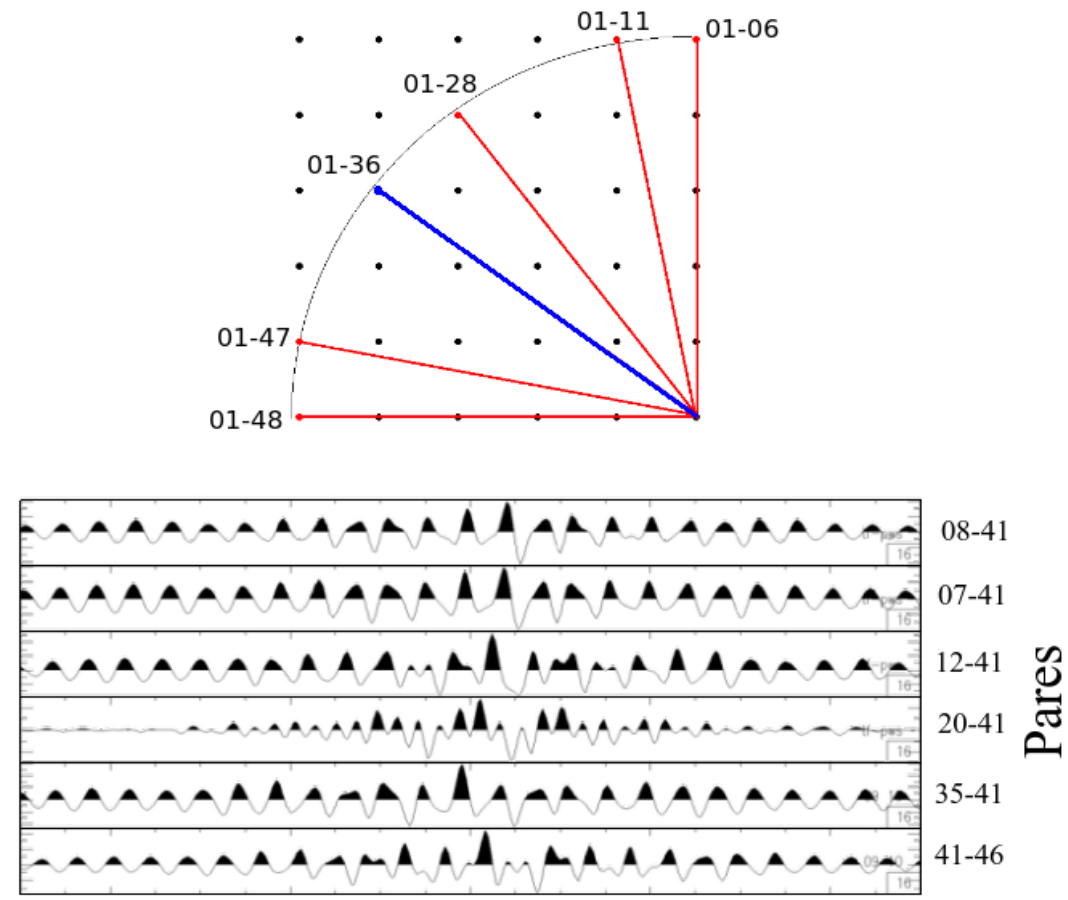

b)

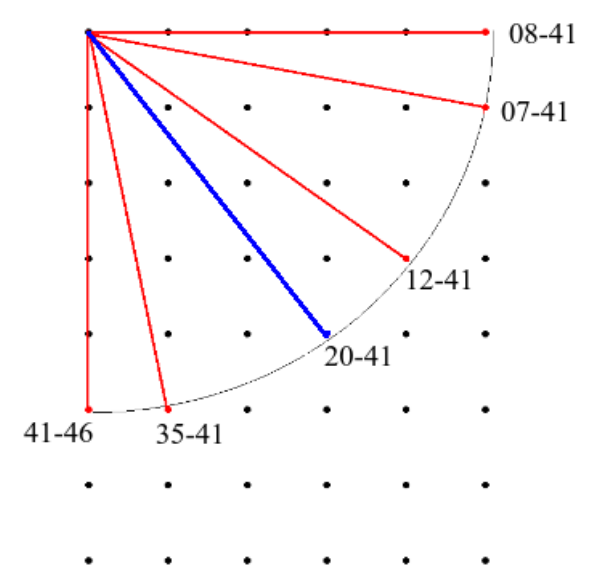

Figura 6: Análise da direção preferencial das fontes de ruído. A distância entre os geofones foi mantida constante em aproximadamente 7,5m. A reta azul indica a direção a qual a relação sinal/ruído é mais baixa. Fata esse que pode ser interpretado como um faixa de predominância do ruido ambiental sobre o ruído coerente. 


\section{Conclusões}

A aplicação rasa do método ANT se torna inviável se na área do levantamento existir alguma fonte de ruído coerente de alta energia, pois este mascara o sinal do ruído ambiental. Como foi o exemplo do gerador que foi acionado próximo de nossa área de estudo.

Observou-se uma dependência da capacidade de recuperação do sinal com a distância, o que pode ser uma limitação do tamanho da área a ser adquirida com este método.

Observou-se também uma dependência com a direção sendo recuperados sinais mais claros para os pares alinhados com as prováveis fontes de ruído.

Observou-se que é necessário o empilhamento de pelo 100 medidas para se obter um sinal adequado. Este valo é significativamente maior do que o observado em trabalhos regionais para estudos da crosta. Provavelmente isto é consequência dos ruídos de maior frequência envolvidos na escala trabalhada, os quais não estão presentes em escalas regionais.

Apesar da falta de imagens tomográficas, os resultados foram positivos, aprofundando o estudo da ANT em pequena escala. Os próximos passos serão as análises das curvas de dispersão, para a geração futura de mapas de velocidades de grupo por período.

\section{Agradecimentos}

Ao Laboratório de Geofísica Aplicada (LGA/IG/UnB) pela disponibilização dos equipamentos geofísicos. E ao CNPq pelo apoio financeiro.

\section{Referências}

BENSEN, G. D., RITZWOLLER, M. H., BARMIN, M. P., LEVSHIN, A. L., Lin, F., MOSCHETTI, M. P., SHAPIRO, N. M., Yang, Y. (2007). Processing seismic ambient noise

data to obtain reliable broad-band surface wave dispersion measurements, Geophys. J. Int., 169, p. 1239-1260.

CLAERBOUT, J. F. (1968). Synthesis of a layered medium from acoustic transmission, Geophysics, 33, p. 264-269.

DAVIS, J. C. (1986). Statistics and data analysis in geology. $2^{a}$ ed. John Wiley \& Sons., p. 550.

FERREIRA, M. V. (2013), Estudo do método de tomografia sísmica de ruído ambiental. Trabalho final de graduação em Geofísica, IG/UnB.
NUNZIATA, C., NISCO, G., Panza, G. F. (2009). S-waves profiles from noise cross correlation at small scale. Engineering Geology, v. 105, n. 3-4, 11, p. 161-170.

ROCHA, M. P.; SEIMETZ, E. X.; BORGES, W. R.; NOGUEIRA, P. V.; AZEVEDO, P. A.; CAVALCANTI, M. M. (2011), Study of seazonal variation of the $P$-wave velocity and porosity estimation using seismic refraction, $12^{\text {th }}$ International Congress of the SBGf. Rio de Janeiro, Brazil.

SHAPIRO, N. M., CAMPILLO, M., STEHLY, L., RITZWOLLER, M. H. (2005). High resolution surface-wave tomography from ambient seismic noise, Science, 307, p. 1615-1618.

SCHIMMEL, M., (1999). Phase Cross-Correlations: Design, Comparisons, and Applications, Bulletin of the Seismological of America, 89(5), p. 1366-1378.

SCHIMMEL, M., PAULSSEN, H., (1997) . Noise reduction and detection of weak, coherent signal through phase weighted stacks, Geophys J Int, 130, p. 497-505.

WAPENAAR, Kees; DRAGONOV, DEYAN; SNIEDER, ROEL; CAMPMAN, XADER; VERDEL, ARIE, (2010). Tutorial on seismic interferometry: Part 1 - Basic principles and applications, Geophysics, 195-209. 